\title{
Laminar Burning Velocity Measurements over Wide Ranging Temperatures and Pressures
}

\author{
Richard Stone \\ University of Oxford \\ Department of Engineering Science, Parks Road, Oxford OX1 3PJ,UK \\ richard.stone@eng.ox.ac.uk
}

\begin{abstract}
Laminar burning velocity data is useful in its own right, but also important for the validation of kinetic schemes and incorporation into models of turbulent combustion. The constant volume vessel methods for determining laminar burning velocity are particularly useful since they lend themselves to measurements at elevated temperatures and pressures, especially when use is made of the data obtained as the pressure rises during combustion. However, care is needed to ensure an appropriate thermodynamic model is used for data analysis and care is needed to account for the effects of stretch during constant pressure measurements and there is a need to exclude data once cellularity as occurred.

This paper will briefly review the steady and unsteady methods for measuring the laminar burning velocity, but then focus on the use of constant volume vessels. It will be shown that it is possible to reconcile the measurements from both the constant pressure phase and the pressure rise phase so long as appropriate analysis is used and care is taken with data selection.
\end{abstract}

